\title{
Personalidad, estrategias cognitivas y tiempo de Inspección
}

\author{
A. ÄNDRÉS \\ A. VIGIL \\ J. TOUS \\ Universidad de Barcelona
}

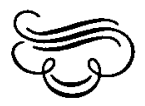

\section{Resumen}

En el presente trabajo se han empleado las medidas cronométricas de Tiempo de Inspección (TI) y Tiempo de Reacción (TR) para estudiar los efectos de las diferencias individuales en extroversión sobre una tarea de discriminación perceptual. Para ello se utilizaron dos grupos de sujetos que habian obtenido puntuaciones máximas y minimas en la dimensión Extroversión del Test EPI de H.J. Eysenck. Se postuló que los extrovertidos obtendrian un TI más corto que los introvertidos y asimismo un TR también más corto, de acuerdo a los modelos de Brebner (1980) y Tous (1986). Los resultados indicaron que si bien existieron diferencias entre extrovertidos e introvertidos en cuanto a las medidas cronométricas, estas diferencias dependieron del uso diferencial de estrategias de resolver la tarea que fueron utilizadas por estos sujetos.

\section{Abstract}

In this paper the chronometric measures, Inspection Time (IT) and Reaction Time (RT) were used to study the effects of individual differences on extroversionsing a perceptual discrimination task. Subjects belonged to the extreme group of introverts $(n=10)$ and extroverts $(n=10)$ as assessed by the Eysenck Personality Inventory. In accordance with Brebner's (1980) and Tous'(1986) model we hypothesized that the extrovert's IT would be sloer than the introvert's, and the extrovert's $R T$ they would be faster than introvert's $R T$. Results indicated the existence of differences in IT and RT between extroverts and introverts, but these differences were related to the differencial use of the response strategy in the tasks used by the groups of subjects.

Agradecimiento. Agradecemos la revisión y comentarios realizados por el Dr. J. M. Tous Ral.

Dirección del autor: Dpto. de Personalidad, Evaluación y Tratamiento Psicológico. Universidad de Barcelona. 


\section{INTRODUCCIÓN}

Con el desarrollo de la Psicología del Procesamiento de Información se ha generalizado el uso de las técnicas cronométricas a ámbitos de la investigación psicológica no estrictamente cognitivos. Asi nos lo muestra la gran proliferación de investigaciones que utilizan el Tiempo de Reacción, el tiempo de Inspección y el Tiempo de Movimiento en el estudio de diferentes aspectos de las Diferencias Individuales tanto en dimensiones de personalidad como en el terreno de las aptitudes y capacidades (A. R. Jensen, 1980a, 1987; J. B. Carroll, 1977; R. J. Sternberg, 1982; H. J. Eysenck, 1982). Esta incorporación de renovadas técnicas experimentales en la investigación psicológica diferencialista en torno a la personalidad ha permitido la reformulación de modelos ya tradicionales como los de H. J. Eysenck o J. A. Gray por autores como J. Brebner o I. Strelau. Nuestro trabajo se enmarca en este enfoque y trata de una de las medidas cronométricas desarrolladas más tardíamente (Vickers et al. 1972; Vickers y Smith, 1986; Brand y Deary, 1982), el Tiempo de Inspección (TI). Este indicador se ha utilizado primordialmente en estudios sobre las capacidades intelectuales (Jensen, A. R., 1980c; Brand y Deary, 1986; Nettelbeck, 1982; Irwin, 1984), sin embargo nosotros lo vamos a utilizar en el estudio de las diferencias individuales en función de la personalidad de los sujetos.

Los primeros desarrollos que se ocupan del Tiempo de Exposición de los estímulos como factor de interés en cuanto a las diferencias individuales psicológicas, corresponden a J. Mc. Cattell (1886). Este autor anticipa la noción de que la duración de una exposición enmascarada de un estímulo es una importante técnica para la medida de las diferencias individuales. J. Mc. Cattell se interesará por el tiempo en que un objeto debe ser observado para poder ser reconocido, desarrollando así el concepto de Tiempo de Percepción. Cattell utilizó para sus mediciones un cronómetro gravitatorio con el cual controlaba el tiempo de exposición mediante una placa deslizante que cubría el estímulo, el tiempo de percepción correspondía a aquel tiempo en el que se realizaba un $90 \%$ de respuestas acertadas y correctas. Con todo ello se anticipó a los actuales conocimientos sobre el enmascaramiento perceptual y el Tiempo de Inspección (TI).

En 1970, D. Vickers desarrolló un modelo de discriminación perceptiva que se conoce con el nombre del «modelo acumulador". Este modelo supone que ante un estímulo el sujeto realiza un muestreo aleatorio del input sensorial, de dimensiones constantes, almacenando estas observaciones hasta que reúne una cantidad de información crítica de evidencia en favor de una respuesta u otra (alternativas), implicando en cada observación un tiempo constante. T. Nettelbeck (1985) señala las principales características de este modelo:

"Vickers asume que la decisión, en un juicio comparativo, es el resultado de un procesamiento de control que transforma la inforamción de un input físico inicial registrándolo en un almacén periférico. El procesamiento se lleva a cabo por extracción de muestras discretas del contenido informativo procedente del registro sensocial; siendo el tiempo invertido en extraer cada muestra, pequeño y constante. Dado que este modelo incorpora la suma como operación básica, Vickers lo denomina modelo acumulador." 
A partir de este planteamiento de D. Vickers el Tiempo de Inspección (TI) quedará definido conceptualmente como el tiempo necesario con el fin de realizar una inspección, es decir la extracción de una muestra discreta de información de un material sensorial registrado periféricamente. Para evaluar empíricamente el TI, y asi operativizar una medida de rendimiento conductual, se utiliza una tarea de discriminación simple, en la cual los tiempos de presentación estimular son muy rápidos y breves. Los estímulos pueden ser de diversos tipos: auditivos, visuales, y táctiles, siendo los más utilizados los visuales. En concreto el paradigma experimental más habitual para la obtención del Tiempo de Inspección consiste en la presentación de dos líneas verticales de diferente longitud, unidas en su parte superior por una raya horizontal siendo la tarea del sujeto el discriminar cual de las dos líneas verticales es de mayor longitud (Vickers y Smith, 1986). Se han utilizado otros tipos de estímulos visuales en diversos trabajos tales como dibujos o nombres de animales, líneas coloreadas, etc. (Vigil y Tous, 1987; Vernon, P. A., 1986). Operacionalmente se considera el Tiempo de Inspección (TI) del sujeto el tiempo mínimo de exposición del estímulo en el que el sujeto discrimina correctamente en un porcentaje considerable de las presentaciones, usualmente superior al 97,5\% (Vickers y Smith, 1986). T. Nettelbeck, en el paradigma de las dos líneas, considera este Tiempo de Inspección es de unos $100 \mathrm{msg}$ para adultos normales y de unos $250 \mathrm{mgs}$ para adultos retrasados mentales (Nettelbeck, 1982).

Como medida cronométrica el TI comparte las características propias de este tipo de técnicas, que se diferencian de las tradicionales medidas psicométricas, según Jensen (1985) en los siguientes aspectos:

1. Propiedades de las escalas de medida; las medidas cronométricas utilizan el tiempo real medido en milisegundos (msg).

2. El milisegundo es obviamente más sensible y preciso a las variaciones conductuales que cualquier otra medida psicométrica.

3. Las técnicas cronométricas son útiles en una amplia gama de poblaciones de sujetos humanos (niños, adultos, retrasados mentales, etc.).

En el estudio de la personalidad el uso de técnicas cronométricas ha sido escaso a través de la historia de la investigación psicológica y menos con el objeto de utilizar los resultados de estas técnicas en el soporte de las teorías de la personalidad. Brebner (1980) opina que ello es debido al interés de la mayoría de los teóricos de la personalidad por la conducta en situaciones interpersonales, dejando un tanto de lado las diferencias individuales que aparecen en las respuestas de los individuos ante estímulos psicológicos no necesariamente sociales. Como excepciones a esta tendencia generalizada, en los estudios de personalidad, podemos citar a autores tales como R. B. Cattell, H. J. Eysenck, J. P. Gray o A. Nebylitzin entre otros autores que se han interesado, la mayor parte de ellos, por la extroversión como rasgo de personalidad. También nosotros en este estudio nos ocuparemos de esta dimensión de personalidad.

H. J. Eysenck(1967) revisa los estudios realizados entre medidas cronométricas y personalidad (principalmente Extroversión y Tiempo de Reacción) concluyendo que la mayoría de los trabajos indican que los extrovertidos poseen un TR más corto y siendo los introvertidos más precisos y cautelosos, aunque por tanto más lentos. D. W. J. Corcoran entre otros, 
ya indicó en su trabajo de 1972 resultados contrapuestos a los resumidos por H. J. Eysenck. Esta contradicción nos permite indicar la existencia de una dependencia del TR no sólo del nivel de extroversión del sujeto sino también, y a la vez, de las características de la tarea. Esta afirmación no resuelve la contradicción de aquellos datos. Diversos autores han pretendido explicar esta dificultad al relacionar TR y Extroversión (Brebner y Cooper, 1974; Brebner, 1980; Tous, 1986). Una de las explicaciones más plausibles y fecundas es la de Brebner y Cooper (1974) que proponen la existencia de dos mecanismos centrales (que actuan en todas las tareas psicológicas, por más simples que estas sean) los cuales son susceptibles de actuar en dos posibles estados: excitación e inhibición. Cada uno de estos estados puede ser elicitado por demandas de análisis del input sensorial, dando lugar a una Excitación Estimular (EE) o a una Inhibición Estimular (IE). Estos estados pueden igualmente estar elicitados por las demandas de organizar la respuesta dando entonces lugar a una Excitación de Respuesta (ER) o a una Inhibición de Respuesta (IR), teniendo en cuenta que el estado general de activación se ve afectado tanto por la estimulación como por las demandas de respuesta.

A partir de este modelo, Brebner sugiere que los extrovertidos producen unos potenciales relativamente grandes de ER para cualquier estado estimular dado, estando pues más «adaptados a responder». Los introvertidos, por el contrario, producen potenciales intensos de EE, por lo que estarían bien «adaptados a explorar». Esta presuposición derivada del modelo de Brebner, modificada por J. Tous en 1986, se ha podido comprobar en un trabajo ya publicado por miembros de nuestro equipo de trabajo (Tous, 1987).

Mientras que en relación a la cronometría mental y la personalidad aún se encuentran algunos trabajos al referirnos al TR y la exţroversión, no ocurre lo mismo al relacionar TI y personalidad. Podemos decir que existe una casi total ausencia de estudios de TI que hayan tenido en cuenta las diferencias individuales en personalidad. Uno de los estudios sobre este tema es el de Brebner de 1986, en el cual se ocupan de estudiar el factor Impulsividad y su efecto en el rendimiento en distintas tareas de TI. M. D. Baguena en 1985, citando a G. Wilson comenta la posibilidad (tan señalada en la actualidad por diversos autores) de que la extroversión pudiese dividirse en dos componentes más elementales: impulsividad y sociabilidad. La impulsividad se entendería como la parte «inestable» de la extroversión siendo este componente el que explicaría, a juicio de esta autora, el deterioro del rendimineto de los extrovertidos en tareas muy monótonas. H. J. Eysenck y M. W. Eysenck (1987) señalan la dificultad en determinar si es la extroversión o la impulsividad el factor más importante en determinar la ejecución conductual.

En el planteamiento de Brebner la impulsividad implicaría una tendencia a responder mayor que la tendencia a inspeccionar o explorar, ello se vería reflejado en la emisión por parte de los extrovertidos de un mayor número de respuestas en ausencia de estímulo, tal y como se ha visto en experiencias anteriores. Por otra parte los extrovertidos tienen un rendimiento menor en tareas que impliquen un análisis estimular elevado, todo ello hace pensar, siguiendo a Brebner, en la posibilidad de utilizar estrategias por parte de los extrovertidos, con el fin de reducir las demandas de 
análisis y optimizar la respuesta. La utilización por parte de algunos individuos de estrategias en tareas de TI fue demostrada por Mackenzie y Bingham (1985). En aquellas tareas la estrategia consistía en utilizar el movimiento aparente en un clásico paradigma de TI (dos líneas verticales de distinta longitud) al variar de posición la más larga a lo largo de los ensayos. Los resultados hallados por Brebner, en el experimento que hemos citado acerca de las relaciones entre extroversión y TI (Brebner y Cooper, 1986), sugieren dos cosas:

«Primero, cualquiera que intente usar el TI como índice libre de las influecnias culturales de la inteligencia debe asegurarse que los errores están producidos por una falta de capacidad discriminativa y no por otros factores y que la tarea no puede ser realizada de diferentes maneras a través de la adopción de diferentes estrategias. En segundo lugar los factores de personalidad, como la Extroversión-introversión pueden jugar un importante papel en las investigaciones mediante el TI.»

La dificultad para encontrar resultados significativos entre los parámetros básicos de procesamiento de información y las diferencias individuales en capacidades básicas, han dado lugar a la sospecha de que otros aspectos más flexibles del funcionamiento cognitivo pueden realizar contribuciones más sustanciales que las habilidades de procesamiento a nivel inferior. Cooper y Regan (1987) señalan como uno de los posibles aspectos a tener en cuenta a las estrategias entendidas como el (los) método(s) que el individuo selecciona para abordar una tarea o resolver un problema.

Para R. J. Sternberg (1987) la elección de una estrategia y su ejecución representa la manifestación clave de la conducta inteligente; para este autor existen propiedades emergentes de las estrategias que no son tan sólo colecciones de procesos, diferenciando el conjunto de la suma de sus partes, y parte del lugar del funcionamiento inteligente reside en ese conjunto. 'Tous (1986) describe las estrategias como' un elemento que dota de mayor flexibilidad al procesamiento en general, encontrándose en la base del procesamiento paralelo, lo que hace modificar la forma secuencial del procesamiento serial, conllevando ello una mejora del tiempo total empleado en una actividad compleja determinada. En opinión de este mismo autor las estrategias se elaboran por el ser humano y por consiguiente no pueden considerarse como los programas que se añaden desde el exterior a las máquinas; no son meras formas de resolver problemas, en algunas ocasiones constituyen formas que impiden la resolución lógica de los problemas, por lo que tampoco pueden ser reducibles a inteligencia, al raciocinio o al pensamiento (Tous, 1986).

J. Baron (1978) ha mencionado varias fuentes que probablemente son causa de diferencias individuales de inteligencia; este autor diferencia entre las capacidades o limitaciones inmodificables del procesamiento de la información y, estrategias o procedimientos modificables para organizar procesos cognitivos a fin de adquirir conocimientos y resolver problemas. El mayor volumen de investigaciones en el campo de las estrategias y diferencias individuales ha sido llevado a cabo por L. A. Cooper y colaboradores (Podgorny, Regan, Glushko y otros). Esta autora ha centrado sus investigaciones en la determinación de las estrategias de los sujetos para resolver tareas de tipo espacial. Los resultados de numerosos trabajos de este grupo 
han llevado a la conclusión de que los sujetos utilizan, para tareas iguales de comparación visual, estrategias diferentes: estrategias analíticas vs. globales. Para esta autora las diferencias pueden deberse a que los sujetos difieran en la forma de codificar y representar patrones visuales en la memoria así como en las representaciones sobre las que actuan las estrategias de comparación (Cooper y Regan, 1987).

En base a los datos disponibles, parecen existir motivos fundamentados para creer que hay factores de personalidad, como la extroversión, que pueden afectar de forma significativa a las medidas cronométricas. Brebner y Cooper (1986) plantean el resultado diferencial que produce la utilización de estrategias en introvertidos y extrovertidos. Cooper y Regan, 1987; J. M. Tous, 1987, y Andrés Pueyo y J. Clua, 1988, plantean la dificultad que existe en inducir la utilización diferencial de estrategias para determinadas tareas en extrovertidos e introvertidos y así poder proceder a la realización de comparaciones para observar las diferencias individuales o grupales en la realización de tareas cognitivas. nuestra opción consiste en presentar una situación experimental de forma tal que favorezca las tendencias individuales de un tipo de procesamiento (Tous, 1986) y así se fuerza a la adopción, por parte del sujeto, de un tipo de estrategia determinada. Este planteamineto ha sido utilizado con anterioridad por M. W. Eysenck (1979) investigando tareas de busqueda visual en la Memoria a Corto Plazo, y también ha sido utilizado por Andrés y Clua (1988) al estudiar las diferencias individuales en reactividad cardíaca en tareas atencionales.

Planteamos en este trabajo las siguientes hipótesis referidas a las diferencias entre extrovertidos e introvertidos en cuanto a: a) uso de Estrategias, b) Tiempo de Reacción y c) Tiempo de Inspección.

Hipótesis A. Estrategias: Si presentamos la tarea de forma que el tipo de presentación de estímulos favorezca la adopción de estrategias, entonces observaremos diferencias significativas en el empleo de las mismas por parte de introvertidos y extrovertidos.

Hipótesis $B$. Tiempo de reacción: Si los sujetos extrovertidos se caracterizan por una elevada excitación de la respuesta, entonces mostraran TR más cortos que los sujetos introvertidos.

Hipótesis C. Tiempo de Inspección: Si los extrovertidos se caracterizan por una mayor inhibición de los procesos centrales de análisis estimular (IE), entonces mostraran un Tiempo de Inspección más corto que los sujetos introvertidos dado que la inhibición estimular (IE) actuará como un atenuador del análisis estimular, reduciendo el TI.

\section{MÉTODO}

\section{Sujetos}

A partir de un grupo de 90 sujetos, todos ellos estudiantes de la Facultad de Filosofía y Letras de Tarragona que contestaron el test EPI de H. J. Eysenck se clasificaron, según su puntuación en el mencionado test a 10 sujetos, todos ellos mujeres, cinco de las cuales habían obtenido en el factor Extroversión una puntuación superior al centil 75 y cinco que habían obtenido una puntuación inferior al centil 25. El factor Neuroticismo os- 
ciló, para la muestra estratificada entre el centil 40 y 60 en ambos grupos. La media de edad de los sujetos fue de 19,3 años ( $\operatorname{máx}=23$, mín $=18$ ).

\section{Aparatos}

Para la presentación visual de los estímulos se utilizó un monitor de fósforo verde Amstrad, modelo GT-65 conectado a un ordenador, mod. CPC-6128 que controló, mediante un programa diseñado al efecto, la presentación de estímulos y la aleatorización de las condiciones. El programa se diseñó para simular la presentación taquistoscópica de los estímulos. Las respuestas de Tiempo de Reacción se registraron mediante un Joystick digital de tarjeta Junjel (mod. RPF-22) y se calcularon las latencias de respuestas y el número de aciertos y errores que cometían los sujetos.

Además del cuestionario EPI se utilizaron el Test de Raven para controlar el CI de los sujetos y un cuestionario de auto-informe, diseñado al efecto, en que se solicitaba al sujeto algunos datos sobre la ingesta de alcohol, café o tabaco antes del experimento. Asimismo en el cuestionario se le solicitaba al sujeto que describiera que estrategia, si la había utilizado, para resolver a la tarea experimental propuesta.

\section{Procedimiento}

La tarea consistió en responder - mediante un dispositivo formado por cuatro sensores digitales dispuestos perpendicularmente en forma de cruz (Joystick RPF-22) — a los estímulos visuales presentados en la pantalla del monitor. Los estímulos fueron cuatro figuras geométricas (triángulo, rombo, cuadrado y hexágono) presentadas en forma de cruz. Cada figura tenía una superficie aprox. de $75 \mathrm{~mm}^{2}$ y con una separación diagonal entre los mismos de $300 \mathrm{~mm}$. En la aparte inferior de la pantalla, y con una separación respecto a la figura más cercana de $125 \mathrm{~mm}$ aparecía el nombre (en letras mayúsculas) de una de las figuras representadas. Todo este complejo estimular aparecía en el centro de la pantalla del monitor.

La tarea del sujeto consistió en accionar el pulsador que coincidía con la posición espacial (arriba, abajo, derecha o izquierda) con la figura cuyo . nombre aparecía en la parte inferior de la pantalla. Como estímulo aviso se utilizó una señal auditiva que aparecía $20 \mathrm{msg}$ antes del complejo estimular. En la consigna experimental se le solicitaba al sujeto que contestara lo más rápidamente posible pero evitando las equivocaciones.

El experimento constaba de tres condiciones que afectaban al tipo de presentación de los estímulos y de correspondencia entre las figuras geométricas y su denominación. En cada condición se.realizaban 260 ensayos sucesivos para cada tipo de presentación. Los primeros 20 ensayos se consideraron de práctica y no se registraron. Los restantes 240 ensayos estaban distribuidos en seis condiciones distintas de tiempo de presentación y fueron: 1) $66 \mathrm{msg}, 2) 116 \mathrm{msg}, 3) 166 \mathrm{msg}, 4) 216 \mathrm{msg}, 5) 266 \mathrm{msg}$ y 6) $316 \mathrm{msg}$. El orden de presentación de estas seis condicioenes fue aleatoria. El intervalo entre ensayos fue fijo y de una duración de $170 \mathrm{msg}$.

En la primera condición, Tipo de Presentación estimular (A) las cuatro figuras geométricas, así como el nombre de una de ellas aparecían en el mismo color gris oscuro. En la segunda condición (B) aparecían dos figuras en tonalidad gris clara y las otras dos oscuras. El nombre que aparecía en 
la parte inferior de la pantalla se presentaba en tonalidad oscura y correspondía siempre a una de las dos figuras presentadas en tono más claro. Y por fin la tercera condición (C) tan solo una figura era presentada en tono claro, figura que se correspondía con el nombre presentado en la parte inferior, que también se presentaba en la misma tonalidad clara.

Entre cada condición de Tipo de Presentación de los estímulos se concedió a los sujetos un intervalo de 5 min. Una vez finalizada la ejecución de todo el experimento los sujetos contestaban a las preguntas del auto-informe antes mencionado. El interés particular de este cuestionario era conocer el uso de algún tipo de estrategia (y cual era esta) para responder en las diferentes condiciones de la tarea experimental. Así pues en cuanto a la tarea experimental se utilizaron dos variables. Tipo de Presentación (A, B y C) y Tiempo de Exposición de los mismos.

En cuanto a las respuestas del sujeto estudiamos el Porcentaje de respuestas correctas, el Tiempo de Reacción, el Tiempo de Inspección y la Estrategia de respuesta utilizada por los sujetos en la resolución de la tarea. Este último dato se obtuvo a partir del cuestionario de autoinforme y simplemente nos interesaba conocer si había utilizado o no alguna estrategia en las distintas condiciones de presentación de los estímulos.

El Tiempo de Inspección se definió operacionalmente, y así se calculó, como el mínimo tiempo de exposición de los estímulos en el cual el sujeto respondía correctamente al $90 \%$ o más de los ensayos.

Estas variables de respuesta y su combinación con las de presentación de los estímulos nos llevarán a analizar los resultados mediante dos diseños distintos, el primero con respecto a las medidas de TR y el segundo respecto al TI. El diseño de análisis de resultados de TR fue un factorial mixto (entre-intra) $2 \times 3 \times 6$ (Personalidad $\times$ Tipo de Presentación $\times$ Tiempo de Exposición) de medidas repetidas. Para el análisis del TI utilizamos un diseño factorial mixto (entre-intra) $2 \times 3$ (Personalidad $\times$ Tipo de Presentación) de medidas repetidas.

En cuanto al resto de variables que se sabe afectan a los estudios de rendimiento en tareas de discriminación perceptual y personalidad fueron controladas: sexo, hora del día, inteligencia, ingesta de sustancias estimulantes/sedantes, post-imágenes, ruido y fatiga.

\section{RESULTADOS}

\section{Estrategias de respuesta}

Los resultados obtenidos a partir de los cuestionarios de autoinforme nos muestran que los sujetos introvertidos y extrovertidos utilizaron la estrategia de los indicios por la tonalidad de las figuras y el nombre de las mismas para decidir su respuesta en la condición C, es decir cuando existía una correspondencia fija entre el tono de la figura y el nombre de la misma. Asimismo los introvertidos y los extrovertidos NO utilizaron ninguna estrategia de respuesta en la condición A. Sin embargo si existió una diferencia en el uso de la estrategia entre extrovertidos e introvertidos en la condición $\mathrm{B}$, cuando la mitad de las cuatro figuras presentadas eran del mismo tono que el estímulo verbal (nombre de la fiugura), en esta condición el $80 \%$ de los extrovertidos utilizaron la tonalidad como indicio para decidir 
su respuesta mientras que ningún introvertido manifestó haber utilizado estrategias de respuesta (ver Tabla I).

\section{TABLA I}

Porcentaje del uso de estrategias de respuesta en las distintas condiciones de presentación de los estimulos

\begin{tabular}{lcccc}
\hline & \multicolumn{3}{c}{ Tipo Presentación } \\
\cline { 2 - 4 } & $\%$ & $\mathbf{A}$ & B & C \\
\hline Extrov. $(s s=5)$ & 0 & 80 & 100 \\
Introv. $(s s=5)$ & 0 & 0 & 100 \\
\hline
\end{tabular}

\section{Tiempo de reacción}

Las latencias de las respuestas correctas de los sujetos en milisegundos aparecen en la Tabla II y en la Figura n. 1.

\section{TABLA II}

Promedios de Tiempo de Reacción de los grupos de sujetos en función de (A). Tipo de Presentación y (B) Tiempo de Presentación de los estímulos

\begin{tabular}{lccc}
\hline & \multicolumn{3}{c}{ Tipo Presentación } \\
\cline { 2 - 4 } \multicolumn{1}{c}{ mseg } & $\mathrm{A}$ & $\mathrm{B}$ & $\mathrm{C}$ \\
\hline Extrov. (ss $=5$ ) & 924.21 & 944.01 & 306.33 \\
Introv. (ss $=5)$ & $(110.70)$ & $(81.32)$ & $(108.71)$ \\
& 977.13 & 1028.14 & 401.47 \\
& $(235.23)$ & $(170.46)$ & $(142.24)$ \\
\hline
\end{tabular}

(A)

\begin{tabular}{lcccccc}
\hline & \multicolumn{7}{c}{ Tipo Presentación } \\
\cline { 2 - 7 } & $\mathbf{6 6}$ & 116 & $\mathbf{1 6 6}$ & $\mathbf{2 1 6}$ & $\mathbf{2 6 6}$ & $\mathbf{3 1 6}$ \\
\hline Extrov. (ss = 5) & 802.33 & 764.75 & 705.83 & 683.92 & 651.25 & 651.25 \\
Introv. (ss = 5) & $(295.44)$ & $(295.01)$ & $(506.47)$ & $(554.95)$ & $(554.46)$ & $(554.15)$ \\
& 871.14 & 899.73 & 842.27 & 779.13 & 720.60 & 690.13 \\
& $(515.41)$ & $(565.56)$ & $(527.75)$ & $(527.75)$ & $(524.94)$ & $(549.72)$ \\
\hline
\end{tabular}

(Datos en mseg)

El porcentaje de respuestas correctas.fue similar al comparar los grupos extrovertidos $(85,97 \%)$ y los introvertidos $(87,67 \%)$ ya que su diferencia no resultó significativa.

Los resultados de TR fueron analizados en un AVAR $(2 \times 3 \times 6)$ y siendo significativos los efectos principales de la variable Personalidad $(F=11,3 ; p<0,001)$, tipo de Presentación $(F=6,1 ; p<0,001)$ y Tiempo de Exposición $(\mathrm{F}=316,01 ; \mathrm{p}<0,001)$. En cuanto a las distintas interacciones debemos decir que en todos los casos no son significativas. 


\section{4}

Figura I

Tiemp. Reacción (mseg.)

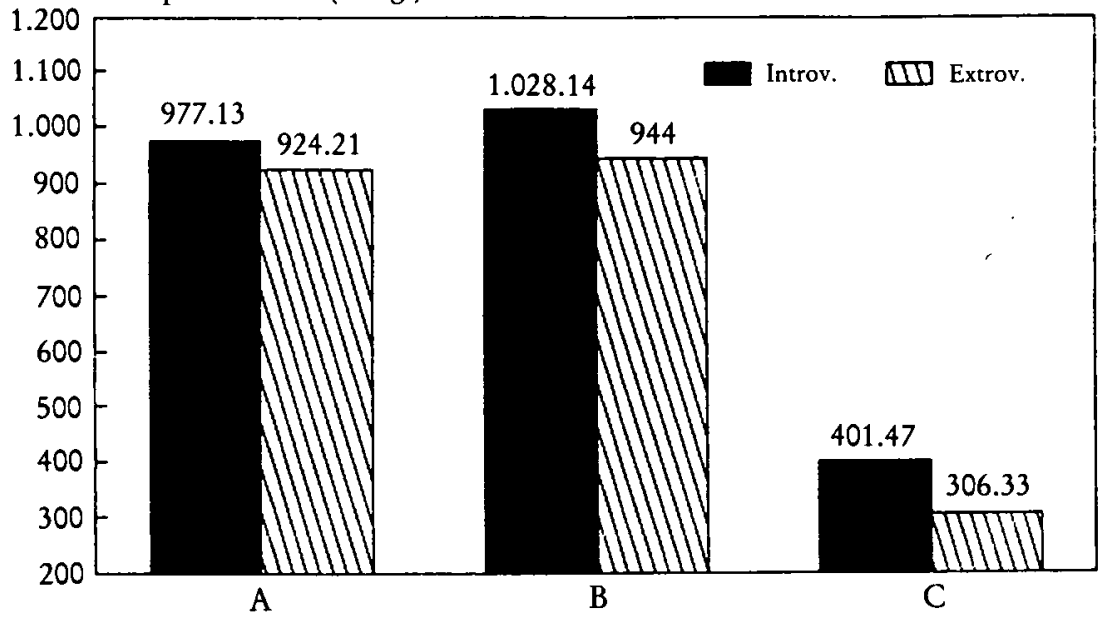

Personalidad y TR Person. X Tipo Present.

Promedios de Tiempo de Reacción obtenidos por los grupos de sujetos Extrovertidos $e$ Introvertidos en las distintas condiciones de presentación de los estímulos.

Tiemp. Inspecc. (mseg.)

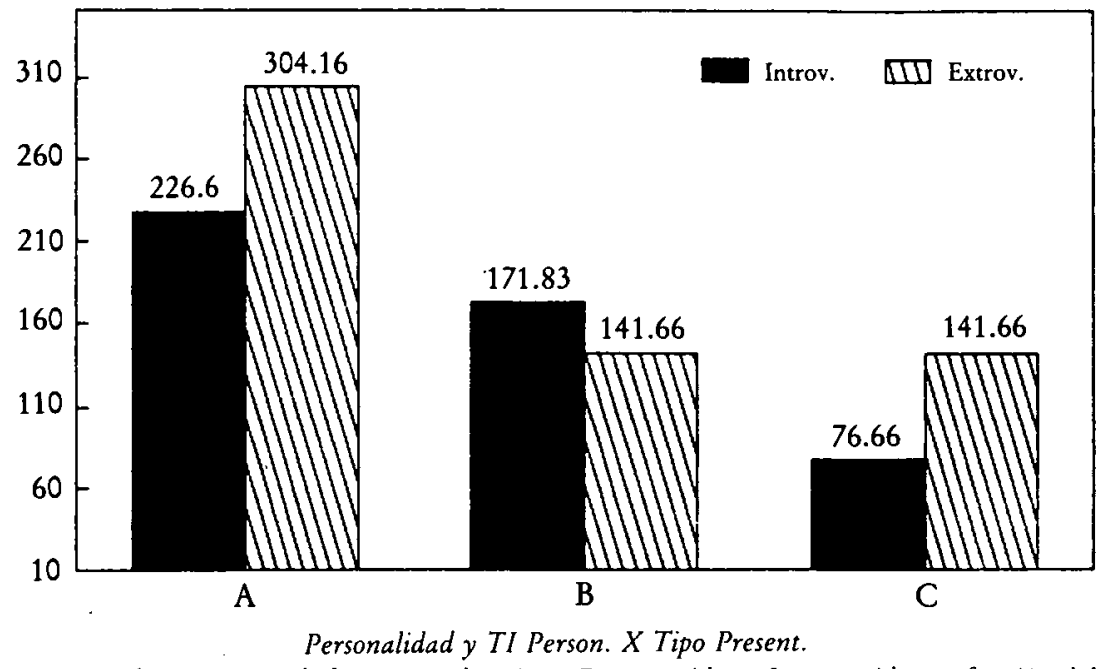

Tiempo de Inspección de los grupos de sujetos Extrovertidos e Introvertidos en función del Tipo de Presentación de los estímulos.

\section{Tiempo de Inspección}

En cuanto a los resultados de TI y de acuerdo al procedimiento de cálculo operacional que hemos indicado anteriormente aparecen indicados en la Tabla n..$^{\circ}$ III.

El AVAR $(2 \times 3)$ realizado sobre estas puntuaciones ofreció una significación de los efectos principales de las variables Personalidad $(F=12,6$; $\mathrm{p}<0,001)$ y Tipo de Presentación $(\mathrm{F}=77,2 ; \mathrm{p}<0,001)$. En cuanto a la 
FiguRA III

condición presentación $A$
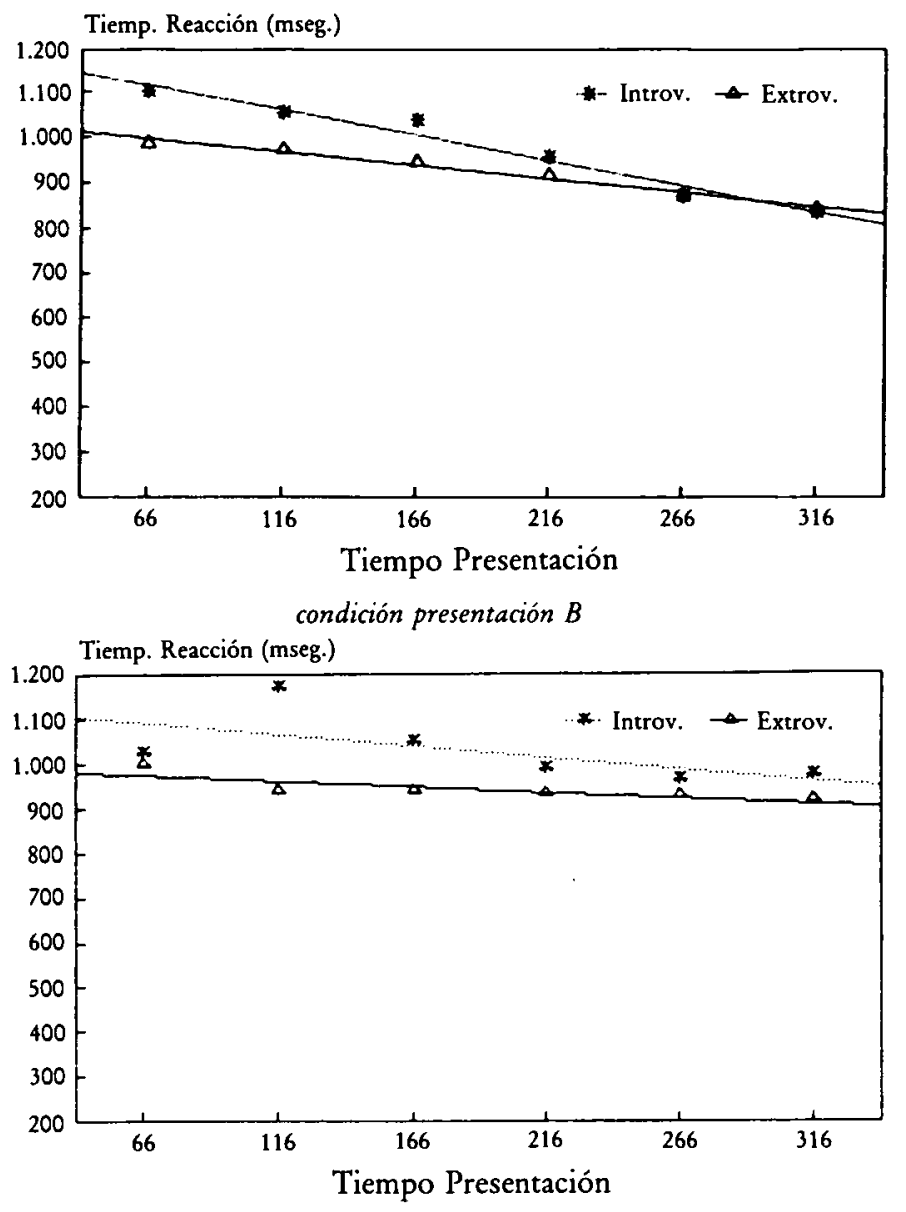

condición presentación $C$

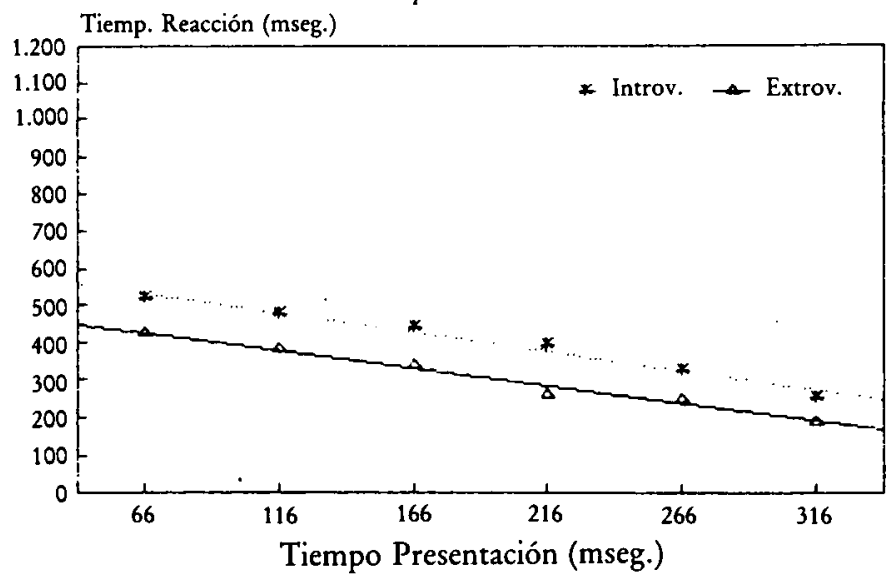

Person. X Tipo Presen. X TR.

Tiempos de Reacción obtenidos por los grupos de sujetos en cada condición de presentación de los estímulos y en función de los tiempos de exposición. 
TABLA III

Valores medios de Tiempo de Inspección en mseg, obtenidos por los grupos de sujetos extrovertidos e introvertidos en las distintas condicciones de Presentación de los estímulos

\begin{tabular}{lccc}
\hline & \multicolumn{3}{c}{ Tipo Presentación } \\
\cline { 2 - 4 }$(\mathrm{mseg})$ & $\mathrm{A}$ & $\mathrm{B}$ & $\mathrm{C}$ \\
\hline Extrov. $(\mathrm{ss}=5)$ & 304.16 & 141.66 & 141.66 \\
Introv. $(s \mathrm{ss}=5)$ & 226.60 & 171.83 & 76.66 \\
\hline
\end{tabular}

interacción Personalidad $\times$ Tipo de Presentación observamos también una notable significación $(F=10,3 ; \mathrm{p}<0,001)$. En la Figura n. ${ }^{\circ} 2$ se pueden observar representados estos resultados.

\section{DISCUSIÓN}

\section{Estrategias: empleo y utilización diferencial}

En base a los resultados obtenidos es plausible afirmar que las condiciones estimulares presentadas han favorecido el empleo de estrategias por parte de los sujetos, estrategias que, aparentemente, en función a los datos disponibles de los autoinformes, se han producido en la dirección por nosotros esperada. Al mismo tiempo, cabe señalar la utilización diferencial de las mismas por parte de los sujetos extrovertidos e introvertidos. Esta utilización diferencial parece confirmar la primera hipótesis formulada en este trabajo. De igual modo parece existir una mayor tendencia en los extrovertidos por emplear estrategias para la realización de tareas cognitivas, tendencia que alcanza su máxima expresión en el segundo tipo de presentación (B), condición en la que 4 de los 5 sujetos extrovertidos manifiestan haber utilizado una estrategia (basada en la tonalidad de las figuras), mientras que ninguno de los sujetos introvertidos manifiesta el empleo de la misma en la condición $B$.

Todo ello nos lleva a suponer que los extrovertidos realizan una reducción de las exigencias del análisis estimular basada en el empleo de estrategias, con el fin de mantener un rendimiento aceptable ante en aumento de la inhibición estimular producida en ellos por la tarea. Esta reducción de las exigencias del análisis estimular no sería tan necesaria en los sujetos introvertidos, dado que los potenciales de excitación estimular relativamente potentes, que según Brebner (1980) poseen los introvertidos permitirían mantener un rendimiento aceptable sin necesidad de recurrir a las estrategias.

\section{Tiempo de Reacción}

El análisis de varianza realizado muestra que tanto el Tiempo de Exposición, como el Tipo de Presentación y la Personalidad influyen significativamente en el TR, sin embargo las interacciones de dos vías entre estas variables no son significativas, por lo que podemos suponer que estas variables están afectando a etapas de procesamiento diferentes, produciendo efectos independientes entre sí que afecten al TR global (ver Tous, 1986). 
Sin embargo en la primera condición de presentación parece existir una leve interacción (ver Fig. 3) debida a la mayor pendiente de la curva del TR en los introvertidos al aumentar la duración del Tiempo de Exposición, ello podría venir explicado por la reducción del TR de los introvertidos cuando las condiciones de la tarea favorecen la discriminación de los estímulos, lo que permite que el criterio de respuesta de los introvertidos, usualmente más cauto (M. W. Eysenck, 1985) que el de los extrovertidos, les permita desidir y ejecutar la respuesta con mayor rapidez.

La hipótesis según la cual los extrovertidos obtendrían unos TR's más cortos ha quedado verificada (ver Fig. 1). Así pues podemos decir que se corrobora la existencia de unos potenciales relativamente grandes de excitación de respuesta (ER) en los extrovertidos.

\section{Tiempo de Inspección}

Los resultados relativos al TI muestran una vez más la necesidad de tener en cuenta las variables de personalidad al utilizar este tipo de técnicas cronométricas. En nuestro caso la variable extroversión-introversión afecta significativamente al TI, del mismo modo que éste se ve afectado por el Tipo de Presentación de los estímulos. Es de gran interés el hecho que entre la Personalidad y el Tipo de Presentación se produce una interacción significativa, ello nos indica que las diferencias observadas en TI para cada Tipo de Presentación varían en función de la personalidad del sujeto. Los datos obtenidos contrastan con la hipótesis de que los extrovertidos obtendran un TI más corto, tanto en la primera condición de presentación de estímulos (Cond. A) como en la tercera (Cond. C) el TI de los introvertidos es más corto que el de los extrovertidos (ver Fig. 1), el hecho de que los extrovertidos presenten un TI más corto en la segunda condición (B) vendría explicado por la utilización de las estrategias, estrategias que no manifiestan utilizar los introvertidos. Sin embargo, también se podrían explicar estos resultados de una forma alternativa, quizás arriesgada pero plausible a la luz de los datos de TI y de TR combinadas, y es que entre introvertidos y extrovertidos en la condición $\mathrm{C}$ ha existido un uso diferencial de estadios de procesamiento; así mientras que los extrovertidos se han limitado a realizar una tarea de búsqueda visual los introvertidos han realizado una tarea de memoria inmediata para verificar lo acertado de sus respuestas (ver Figs. 1 y 3 ).

Finalmente no se ha podido contrastar la hipótesis relativa a los resultados obtenidos al comparar dentro de los grupos de sujetos, tanto extrovertidos como introvertidos, los que utilizaron estrategia frente a los que no la usaron, dado que dentro de las condiciones de presentación $\mathrm{A}, \mathrm{B}$ y C, los individuos no presentan diferencias en el empleo o no de estrategias - salvo 1 extrovertido en la condición B- lo cual nos impidió cọntrastar la mencionada hipótesis.

Para finalizar esta discusión deseamos abordar dos nuevos temas: los estadios intermedios de procesamiento y las relaciones entre TR y TI en introvertidos y extrovertidos. Si consideramos que en los estadios del procesamiento de información, existe unprimer estadio de impresión y un último estadio de expresión, con una serie de estadios intermedios indeterminados (Tous, 1986; S. Sterbnerg, 1969), podemos suponer que los individuos introvertidos han empleado en el tercer Tipo de Presentación de los 
estímulos (C), estadios que no han sido utilizados $\multimap$ lo han sido en menor medida - por los sujetos extrovertidos, es factible que este(os) estadio(s) se utilizaron en relación a los procesos de búsqueda en la MCP y de verificación de respuesta, que son estadios intermedios de procesamiento, entre los de impresión y expresión. Esta sugerencia se plantea tan solo de forma hipótetica y se sustenta en la comparación de los valores absolutos de TR en introvertidos y extrovertidos con los resultados hallados en tareas de búsqueda visual y recuerdo inmediato por autores ya clásicos como M. I. Posner (1978) S. Sternberg (1969) y Shiffrin y Schneider (1977).

Finalmente se han observado diferencias notables en la relación entre TR y TI al comparar estas relaciones para introvertidos y extrovertidos. Los sujetos introvertidos, a través de los Tipos de Presentación de los estímulos, presentan un descenso progresivo de los TR's (Fig. 2) así como de los TI's (Fig. 1), aun menor TI le corresponde un menor TR, de hecho obtuvimos una correlación entre TR y TI para los introvertidos de $r=70$ $(p<0,001)$. Sin embargo en el caso de los sujetos extrovertidos, no se ha dado dicha correlación entre TI y TR $(r=10$, n.s.). Esto nos permite aportar una prueba más en favor de que TI y TR - como mínimo en el caso de los extrovertidos- nos permiten obtener indicadores de distintos estadios de procesamiento.

\section{CONCLUSIÓN}

Si como parece demostrarse, las variables de personalidad afectan al TI, creemos necesario un mayor análisis tanto de las influencias de dimensión introversión-extroversión, como de otras dimensiones de la personalidad. $\mathrm{Al}$ referirnos al problema del uso diferencial de las estrategias cabe preguntarse si la tendencia a desarrollar estas, en base a las tendencias de conducta de los sujetos (personalidad) no sería el resultado de una mayor adaptación a situaciones nuevas, característica que H. J. Eysenck y M. W. Eysenck (1987) citan como propia de los extrovertidos. Ello nos lleva a preguntarnos si los datos obtenidos son consecuencia de un factor de adaptabilidad a nuevas situaciones, o bien si adaptarse a situaciones nuevas es consecuencia del empleo de estrategias, que dotan de mayor flexibilidad al procesamiento general, mediante lo que J. M. Tous (1986) define como el establecimiento de una nueva ordenación secuencial de los pasos necesarios para la consecución de un resultado correcto y eficaz.

\section{Referencias}

ANDRÉs PUEYo, A. y CluA, F. X. (1988). Individual differences in attentional perfomance and cardiac reactivity. Psychopbysiology (in press).

ARnau, J. (1982). Teoria de la detección de señales. Barcelona: Ed. E. Univ. Barcelona.

BAgÜENA, M. J. y BelloCH, A. (1985). Extroversión, Psicoticismo y dimensiones emocionales de la personalidad. Valencia: Promolibro.

BARON, J. (1978). Intelligence and general strategies. En G. Underwood (comp.): Strategies in information processing. Londres: Academic Press.

BRAND, C. R., y DEARY, I. J. (1982). Intelligence and inspection time. En H.J. Eysenck (comp.): A model of intelligence. Nueva York: Springer Verlag.

BREBNER, J., y COOPER, C. (1974). The effect of a low rate of regular signals upon the reaction time of introvert and extraverts. Jour of Research in Personality, 8, 263-276. 
BREBNER, J. (1980). Reaction time in personality theory. En A. T. Welford (comp.): Reaction time. Londres: Comedia Press.

Brebner, J., y COOPER, C. (1986). Personality factors and inspection time. Person. and Indiv. Diff. 7, 5, 709-714.

CARROLL, J. B. (1977). How shall we study individual differences in the cognitive abilities? Intelligence, 2, 87-115.

CATTELL, J. Mc. (1886). The inertia of the eye and the brain. Brain, 8, 295-318.

COOPER, L., y REGAN, D. T. (1987). Atención, percepción e inteligencia. En R. J. Sternberg (comp.): Inteligencia humana. Vol. 2. Barcelona: Paidós.

CORCORAN, D. W. J. (1972). Studies of individual differences at the applied psychology unit. En V. D. Nebylitzin y A. Gray (comps.): Biological basis of individual bebavior. Londres: Academic Press.

DEARY, I. J. (1986). Inspection time: discovery or rediscovery? Person. and Indiv. Diff. 7, 5, 625-631.

EYSENCK, H. J. (1967). The biological basis of personality. Thomas, Springfield.

EYSENCK, H. J. (1986). Inspection time and intelligence: a historical introduction. Person. and Indiv. Diff. 7, 5, 603-607.

EYSENCK, H. J., y EYSENCK, M. W. (1987). Personalidad y diferencias individuales. Madrid: Pirámide.

EYSENCK, M. W., y EYSENCK, M. C. (1979). Memory scanning, introversion-extroversion and levels of processing. Jour. of Res. in Personality, 13, 305-315.

IRWIN, R. J. (1984). Inspection time and its relation to intelligence. Intelligence, 8, 47-65.

JENSEN, A. R. (1980a). Chronometric study of mental abilities. En A. T. Welford (comp.): Reaction time. Londres: Comedia Press.

JENSEN, A. R. (1980b). Bias in metal testing. Londres: Methuen Co.

JENSEN, A. R. (1980c). Chronometric analysis of intelligence. Jour. of Soc. Biol. Struct., 3, 102-122.

JENSEN, A. R. (1985). Methodological and statistical techniques for the chronometric study of mental abilities. En C. R. Reynolds y V. L. Wilson (comps.): Methodological and statistical advances in the study of individual differences. London: Plenum Press.

JENSEN, A. R. (1987). Process differences and individual differences in cognitive tasks. Intelligence (in press).

MARIE-PAUL, L., y MUNiz, J. (1987). Inteligencia psicométrica y tiempo de inspección. En Yela, M. (comp.): Estudios de inteligencia y lenguaje. Madrid: Pirámide.

MCKeNZIE, B., y BINGHAM, E. (1985). Inspection time and response strategies in a universitary population. Australian Jour. of Psychology, 37, 257-268.

NETTELBECK, T., y LALLY, M. (1980). Inspection time, intelligence and response strategy. Amer. Jour. of Mental Deficiency, 1, 78-91.

NETTELBECK, T. (1982). Inspection time: an index for intelligence? Quart. Jour. of Exptal. Psychol., 34a, 299-312.

NETTELBECK, T. (1985). Inspection time and mild mental retardation. Intern. Jour. of Res. in Mental Retardation, 13, 109-141.

POSNER, M. I. (1978). Chronometric explorations on mind. Erlbaum: Hillsdale.

SMITH, G. A. (1986). Inspection time and response strategies in a choice response task. Person. and Indiv. Diff. 7, 5, 701-707.

SHIFFRIN, R. M., y SCHNEIDER, W. (1977). Controlled and automatic human information processing, II. Psychol. Rev., 84, 127-190.

STERNBERG, S. (1969). The discovery of processing stages: extensions of Donder's method. Acta Psychol., 30, 276-315.

STERNBERG, R. J. (1982). Recent advances in research on intelligence. Hillsdale: L.E.A.

STERnBerg, R. J. (1987). La inteligencia bumana. Vols. 1 y 2. Barcelona: Paidós.

TEA (1976). Manual del test E.P.I. de H.J. Eysenck. Madrid: TEA.

Tous, J. M. (1978). Psicología experimental: problemas de teoría y método. Barcelona: Omega.

Tous, J. M. (1986). Psicologia de la personalidad. Barcelona: Ed. PPU.

Tous, J. (1987). La personalidad y el método científico natural. Rev. de Psicología Universitas Tarraconensis. Vol. 8 (2).

Vernon, P. A. (1986). Inspection time: does it measure intelligence? Person. and Indiv. Diff. 7, 5, 715-720.

VERNON, P. A. (1986). Intelligence and speed of information processing. Nueva York: Ablex Co.

VIGIL, A., y Tous, J. (1987). Personalidad y tiempo de inspección. Tesis de licenciatura no publ.: Univ. Barcelona.

VICKERS, D. (1970). Evidence for an acumulator model of psychophysical discrimination. Ergonomics, $13,37-58$.

VICKERS, D., y SMITH, P. L. (1986). The rationale for the inspection time index. Person. and Indiv. Diff. 7, 5, 609-623.

Yela, M. (comp.) (1987). Estudios de inteligencia y lenguaje. Madrid: Pirámide. 